\section{Fraud, flaws or failures in scientific publishing}

\author{
Phil Wiffen
}

This journal has strongly advocated the need for pharmacy practitioners to undertake research and publish their findings. Undertaking research and not publishing is considered by some to be research waste and may, in certain circumstances, be unethical. The pressure to publish, which is probably much stronger in other health professions, can lead to perverse situations, resulting in fabrication of data, flaws due to lack of diligence or failures of not being aware of others rights.

Retraction Watch ${ }^{1}$ is a blog that monitors retractions from scientific journals. Top of the leader board is Yoshitaka Fujii with over 180 retracted papers, a number of these (over 20) were so-called studies of granisetron for postoperative nausea and vomiting. The studies were all of a similar size with very similar numbers of efficacy and adverse events (Tramèr ${ }^{2}$ ). The 'studies' claimed a better antiemetic effect than studies published by other authors. If data look too good to be true then they probably are! More concerning is that such data can influence formulary choices and, ultimately, patient care.

Plagiarism-a term invented several centuries ago from the Latin for kidnapper-is by no means new. This crops up from time to time, probably because of a lack of awareness rather than a deliberate attempt to deceive. There are a number of software packages that quickly identify the original sources. The manuscript procedure for EJHP includes a routine plagiarism check.

Honorary authorship and ghost writing are another annoyance in the publishing world. Honorary authorship is where a person in authority (such as a departmental head) insists on having their name

Correspondence to Professor Phil Wiffen, Pain Research Unit, Churchill Hospital, Old Rd, Oxford OX3 7LE, UK; phil.wiffen@ndcn.ox.ac.uk added to the author list without making a direct contribution to a paper. This is an abuse of position. It is more prevalent in some cultures but should be firmly resisted.

Authorship is defined by the International Committee of Medical Journal Editors (ICMJE) ${ }^{3}$ as

'Substantial contributions to the conception or design of the work; or the acquisition, analysis, or interpretation of data for the work; AND

Drafting the work or revising it critically for important intellectual content; AND

Final approval of the version to be published; AND

Agreement to be accountable for all aspects of the work in ensuring that questions related to the accuracy or integrity of any part of the work are appropriately investigated and resolved.'

Ghost authorship is when a paper by unacknowledged authors is written but published under the name of an often wellknown expert who may get rewarded for the privilege. The technique is still occasionally use by Pharma. Neither approach is acceptable to EJHP.

Peer review fraud ${ }^{4}$ is another issue currently raising its head. Some authors have been shown to create names and email addresses that come back to themselves so that they can write a positive review. This journal does ask authors for suggestions of peer referees but editors will only choose one or two of these together with other names of those who have published on similar topics.

Sometimes things just go wrong. There was a case of a Cochrane review back in 2004 where the labels of the meta-analysis were set up the wrong way round so that the answer presented was the total opposite of the true answer. The review was promptly corrected and republished.
Retraction Watch cites several examples of papers retracted owing to the unauthorised use of a scale to assess adherence known as MMAS-8. ${ }^{5}$ The full name is Morisky Medication Adherence Scale. The website ${ }^{6}$ for this tool has little information but carries a warning that permission for use is required. The problem for users is that the tool, which largely asks questions that many pharmacists would ask, is described in some peer review papers without reference to the need for a fee paid permission to use the tool. One author to this journal is currently caught up in an unpleasant situation where the owners of MMAS- 8 are seeking redress. Potential authors need to be aware of this and may need to seek out other tools. A useful summary paper by Lam and Fresco may be helpful.

\section{EAHP Statement 6: Education and Research}

\section{Competing interests None declared.}

Provenance and peer review Commissioned; internally peer reviewed.

(c) European Association of Hospital Pharmacists (unless otherwise stated in the text of the article) 2017. All rights reserved. No commercial use is permitted unless otherwise expressly granted.

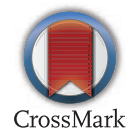

To cite Wiffen P. Eur J Hosp Pharm 2017;24:195.

Eur J Hosp Pharm 2017;24:195.

doi:10.1136/ejhpharm-2017-001316

\section{REFERENCES}

1 Retraction Watch. http://retractionwatch.com/ (accessed June 2017).

2 Tramèr MR. The Fujii story: a chronicle of naive disbelief. Eur J Anaesthesiol 2013;30:195-8.

3 ICMJE. Defining the role of authors and contributors. http://www.icmje.org/recommendations/browse/rolesand-responsibilities/defining-the-role-of-authors-andcontributors.html

4 Haug CJ. Peer-review fraud--hacking the scientific publication process. N Eng/ J Med 2015:373:2393-5.

5 Retraction Watch. http://retractionwatch.com/2017/ 01/26/use-research-tool-without-permission-youllhear/\#more-47897 (accessed June 2017).

6 MMAS Research LLC. http://morisky.org/ (accessed June 2017).

7 Lam WY, Fresco P. Medication Adherence Measures: an Overview. Biomed Res Int 2015;2015:1-12. 